\title{
Beija-flores (Aves: Trochilidae) e seus recursos florais em uma área de caatinga da Chapada Diamantina, Bahia, Brasil
}

\author{
Caio Graco Machado
}

\begin{abstract}
Laboratório de Ornitologia e Mastozoologia, Departamento de Ciências Biológicas, Universidade Estadual de Feira de Santana. Avenida Transnordestina, Novo Horizonte, 44036-900 Feira de Santana, Bahia, Brasil. E-mail: graco@pq.cnpq.br
\end{abstract}

\begin{abstract}
Hummingbirds (Aves: Trochilidae) and their floral resources in an area of caatinga vegetation in the Chapada Diamantina, Bahia State, Northeast Brazil. This study investigated the species of hummingbird occurring in an area of caatinga vegetation, examining their seasonal activities, the assemblages of plants that they used, as well as the floral characteristics and flowering phenology of that vegetation. These surveys were performed in an area of arboreal caatinga in Chapada Diamantina, municipality of Mucugê, state of Bahia, Brazil, during 12 expeditions undertaken on a bi-monthly basis between October, 2005 and August, 2007. Field activities included observations made of the visiting hummingbird species, their behavior, and the frequency of their visits; as well as the plant species visited, their floral attributes, size, and the flowering phenophase evident on the occasion of each expedition. Seven species of hummingbirds were registered, including five residents. Phaethornis pretrei (Lesson \& Delattre, 1839) acted as the organizer of this pollination guild. This species and Chlorostilbon lucidus (Shaw, 1812), these two species were considered the principal pollinators within the community. The hummingbirds visited a total of 29 plant species, of which only 12 are considered ornitophilous. The plant community presented a continuous flowering, with 19 species flowering in both the dry and the rainy season, permitting the year-round permanence of resident hummingbird species.
\end{abstract}

KEY WORDS. Espinhaço range; ornitophily; semi-arid.

Muitas espécies de plantas necessitam de animais vertebrados como agentes polinizadores (BAwA 1990) e frequentemente essa interação associa-se a especializações (STILES 1981) de modo que haja correspondência entre a morfofisiologia floral e a morfologia, o sistema sensorial e o comportamento de forrageio de seu visitante (FAEGRI \& PIJL 1980).

Os beija-flores (Aves: Trochilidae) podem ser responsáveis pela polinização de até 15\% das espécies de plantas das comunidades na Região Neotropical (FeInsinger 1983). No Brasil, a maior parte das pesquisas sobre beija-flores e o conjunto de plantas que eles utilizam se baseia em dados obtidos em curtos períodos (SNow \& Teixeira 1982, Snow \& Snow 1986, Vasconcelos \& Lombardi 2001, Mendonça \& Anjos 2005). São poucos os estudos que contemplam os ciclos sazonais destas comunidades (e.g. Fischer \& Araujo 1995, SAzima et al. 1996, Buzato et al. 2000, Araujo \& Sazima 2003, Kaehler et al. 2005, Leal et al. 2006, Machado \& Semir 2006, Machado et al. 2007).

No nordeste do Brasil são escassos os estudos sobre os beija-flores e seus recursos florais (e.g. Machado \& SAzIma 1995, Machado \& Lopes 2002, 2003, 2004, Leal et al. 2006, Colaço et al. 2006, MACHADO et al. 2007). Na Chapada Diamantina, porção norte da Cadeia do Espinhaço, no Estado da Bahia, apesar da riqueza de tipos vegetacionais, da grande diversidade e do elevado endemismo de espécies vegetais (GiulietTi et al. 1997,
Juncá et al. 2005), as investigações sobre este tipo de interação somente foram conduzidas em áreas de campo rupestre (CoLAço et al. 2006, MACHADO et al. 2007).

Visando ampliar o conhecimento sobre a troquilofauna de ambientes de caatinga, este estudo investigou quais são as espécies de beija-flores que ocorrem em uma área de caatinga na Chapada Diamantina, descrevendo seus padrões sazonais, estratégias de forrageamento e a similaridade do conjunto de espécies de plantas que visitam, registrando, destas, seus atributos florais e prováveis padrões fenológicos de floração.

Adicionalmente, considerando que a acessibilidade ao néctar em flores de corola longa seja desigual aos beija-flores com comprimento de bicos diferentes (Brown \& Bowers 1985, Fenster 1991), este estudo também investigou se há correlação entre os tamanhos de bicos das diferentes espécies de beijaflores com os comprimentos de corola das flores das diferentes espécies de plantas que exploram.

\section{MATERIAL E MÉTODOS}

Este estudo foi desenvolvido em uma a área de caatinga (13 $\left.07^{\prime} 47,6^{\prime \prime} S, 41^{\circ} 35^{\prime} 41,1^{\prime \prime} \mathrm{W}\right)$ localizada na Serra do Bastião, Chapada Diamantina, município de Mucugê, Bahia, entre 900 a $980 \mathrm{~m}$ de altitude. O clima na região é mesotérmico do tipo "Cwb", subtropical moderado úmido, com estações chuvosa 
(outubro a março) e seca (abril a setembro) (STRADMANN 1998). A temperatura média anual é de $22^{\circ} \mathrm{C}$ e a pluviosidade média anual é de aproximadamente 1.300 mm (STRAdmann 1998). A vegetação é decídua, do tipo caatinga arbórea, cujo sub-bosque é denso e o dossel com $5 \mathrm{~m}$, de altura, em média.

De outubro de 2005 a agosto de 2007 foram efetuadas 12 expedições bimestrais com duração de cinco dias cada. Durante as expedições foram realizadas sessões de observações naturalísticas, utilizando-se o método do indivíduo-focal (AltMann 1974) junto às plantas, ornitófilas ou não, cujas flores eram visitadas por beija-flores. A determinação quanto à síndrome de polinização seguiu FAEGRI \& PIJL (1980).

Sempre que possível foram monitoradas, ao mesmo tempo, mais de uma planta, de uma mesma ou de espécies diferentes, totalizando 3412 horas de observações em campo. As sessões de observação se iniciavam ao amanhecer e findavamse ao pôr-do-sol.

Foram registradas todas as espécies de beija-flores avistadas e aquelas que visitavam as flores das plantas monitoradas. Registrou-se, também, se estas aves realizavam visitas legítimas, que são aquelas em que a ave investe pela frente da flor, entrando em contato com suas partes férteis e se impregnando de pólen. As demais visitas foram classificadas como ilegítimas. A identificação dos beija-flores foi efetuada em campo, com auxílio de guia (GRANTSAU 1988) e a nomenclatura taxonômica seguiu as recomendações do Conselho Brasileiro de Registros Ornitológicos (2007).

Quanto ao padrão sazonal, espécies de beija-flores foram categorizadas como residentes, se registradas ao longo do ano todo (mesmo que não avistados em meses não consecutivos), ou não-residentes, quando não avistadas em dois ou mais meses consecutivos.

Também foram registradas as interações agonísticas inter e intra-específicas entre os beija-flores, sendo considerados agonismos as manifestações agressivas, como perseguições ou bicadas, não sendo computadas manifestações sonoras.

Neste estudo, os tipos de estratégias de forrageamento das espécies de beija-flores foram determinados baseados em Feinsiguer \& Colwell (1978), que propõem a estratégia do tipo forrageamento em "linhas de captura" ("traplining") e do tipo territorialista. No primeiro tipo, os beija-flores percorrem circuitos extensos repetidamente, em intervalos de tempo espaçados e regulares. Na estratégia territorialista, os beija-flores defendem continuamente, através de interações agonísticas, territórios restritos e ricos em recursos.

As plantas visitadas pelos beija-flores foram identificadas por especialistas a partir de exsicatas de suas partes férteis, que foram depositadas no Herbário da Universidade Estadual de Feira de Santana (HUEFS). A nomenclatura taxonômica das plantas seguiu a orientação de Missouri Botanical Garden's VAST (2007).

Dentre as espécies de plantas amostradas, foram registrados em campo a coloração da flor e das estruturas anexas, a concentração do néctar (com uso de refratômetro de bolso), o tipo de flor (tubo, pseudo-tubo, goela, campânula, funil, disco, pincel, estandarte ou capítulo - Faegri \& Pijl 1980). Também foi medido o comprimento da corola das flores tubulosas, em goela, campânula e funil com paquímetro (precisão de 0,01 $\mathrm{mm})$. No presente estudo, estas flores foram classificadas em curtas (1 a $15 \mathrm{~mm}$ de comprimento de corola), médias (15 a 30 $\mathrm{mm}$ ) e longas (acima de $30 \mathrm{~mm}$ ).

De cada espécie de planta foram retiradas amostras de néctar de 10 flores (de indivíduos diferentes) com microsseringa graduada e determinada a sua concentração de açúcares hidrossolúveis, utilizando refratômetro de bolso (Atago ${ }^{\circledR} 0 \%$ a 95\%); as tomadas de néctar foram feitas sempre pela manhã, por volta das 09:00 h.

Foram consideradas plantas ornitófilas aquelas cujas flores têm antese diurna, são inodoras, com coloração conspícua das sépalas, pétalas ou de suas estruturas anexas, com a produção de néctar de baixa concentração de açúcares (de 20 a 35\%) (Faegri \& Pijl 1980) e com os nectários afastados das partes férteis, que estão normalmente expostas (FAEGRI \& PIJL 1980).

De cada espécie de planta foi registrado o número de sessões de visitas efetuadas pelos beija-flores. Considerou-se como uma sessão de visita o evento de aproximação de uma ave com forrageamento em uma ou mais flores de uma mesma planta monitorada, até seu afastamento. Para o cálculo da freqüência de visitas das aves às diferentes espécies de plantas, foi considerado o número de sessões de visitas em cada planta por hora de observação.

Para a determinação do padrão de floração são necessários registros mensais das espécies de plantas estudadas (Newstrom et al. 1994); uma vez que as expedições a campo foram bimestrais, fez-se a estimativa do padrão fenológico de floração de cada espécie, registrando-se, em cada expedição, a presença da fenofase de floração nas plantas utilizadas pelos beija-flores que ocorriam na área de estudo. A categorização dos padrões fenológicos de floração estimados seguiu Newstrom et al. (1994).

Para se avaliar o quanto há similaridade no uso das diferentes espécies de plantas pela troquilofauna estudada, foi feita a análise de agrupamento hierárquico pelo método das distâncias médias entre os grupos (UPGMA) (SNEATH \& SoKAL 1973) com o coeficiente de distância métrica Euclidiana das freqüências de visitas em cada uma das diferentes espécies de plantas.

Para investigar se a acessibilidade ao néctar em flores de corola longa é desigual aos beija-flores com comprimento de bicos diferentes, avaliou-se, com o coeficiente de Spearman, a correlação entre o comprimento do bico das espécies de beijaflores estudadas com a média do comprimento da corola das flores de cada uma das espécies de plantas que visitaram. As medidas de comprimento de bicos de beija-flores foram obtidos a partir de animais capturados com redes de neblina na área de estudo, de espécimes da coleção da Divisão de Aves do Museu de Zoologia da Universidade Estadual de Feira de Santana (MZUEFS) e também de registros disponíveis em literatura (Grantsau 1988, Vasconcelos \& Lombardi 2001). 


\section{RESULTADOS}

Foram registradas sete espécies de beija-flores: Anopetia gounellei (Boucard, 1891), Phaethornis pretrei (Lesson \& Delattre, 1839), Eupetomena macroura (Gmelin, 1788), Chrysolampis mosquitus (Linnaeus, 1758), Colibri serrirostris (Vieillot, 1816), Chlorostilbon lucidus (Shaw, 1812) e Amazilia lactea (Lesson, 1832), sendo os dois primeiros Phaethornithinae e os demais, Trochilinae (Tab. I).

Anopetia gounellei foi registrada em todas as expedições, exceto dos meses de outubro de 2005 e fevereiro de 2006. Indivíduos desta espécie forrageavam sempre no sub-bosque, onde visitaram nove espécies de plantas, cinco delas ornitófilas (Tab. II). Os intervalos entre as sessões de visitas foram espaçados, em intervalos de no mínimo 60 minutos e todas as visitas às flores foram legítimas.

Phaethornis pretrei foi registrada em todas as expedições (Tab. I). Indivíduos desta espécie visitaram 18 espécies de plantas (Tab. II), sendo os únicos troquilídeos visitantes de três delas: Alstroemeria rupestris M.C. Assis, Mandevilla scabra Lindau e Augusta longifolia (Spreng.) Rehder, todas com corola longa. Visitaram principalmente espécies ornitófilas (11 espécies) e realizaram sessões de visitas em intervalos de no mínimo 60 minutos, não permanecendo junto aos recursos explorados, estabelecendo e defendendo territórios. Realizaram apenas visitas legítimas às flores.

Eupetomena macroura foi registrada em apenas três expedições (Tab. I), quando indivíduos visitaram apenas duas espécies de plantas, de forma legítima: a entomófila Setilobus simplicifolius (DC.) K. Schum e a ornitófila Pyrostegia venusta (Ker Gawl.) Miers, ambas com corola longa.

Indivíduos de $A$. lactea e de C. mosquitus foram registrados na maioria das expedições realizadas (Tab. I). Os primeiros visitaram flores de 13 espécies de plantas, sendo seis ornitófilas e quase todas com corolas médias ou longas (Tab. II). Indivíduos de C. mosquitus foram registrados forrageando em nove espécies de plantas, incluindo cinco ornitófilas (Tab. II), todas também com corolas médias ou longas. Beija-flores destas espécies realizaram várias visitas ilegítimas às flores de Pleonotoma cf. melioides (S. Moore) A.H. Gentry, perfurando a base da corola para obter o néctar.

Em todas as plantas que exploraram, indivíduos destas duas espécies de troquilídeos realizavam visitas freqüentes, permanecendo, entre uma e outra sessão de forrageio, empoleiradas próximo às plantas floridas, vigiando e defendendo seus territórios.

Indivíduos de $C$. serrirostris ocorreram na área apenas no mês de fevereiro de 2006 (Tab. I), quando visitaram $S$. simplicifolius e Helicteres velutina K. Schum.

Chlorostilbon lucidus foi registrada em todas as expedições. Os beija-flores desta espécie visitaram o maior número de plantas na área estudada (Tab. I), explorando flores de 21 espécies, das quais apenas sete são ornitófilas (Tab. II); esta espécie foi a visitante exclusiva de seis espécies de plantas - Dasyphyllum candolleanum (Gardner) Cabrera, P. cf. melioides, Chaetocalyx subulatus Mackinder, Lafoensia glyptocarpa Koehne, Lantana camara Linnaeus e Stachitarpheta quadrangula Nees \& Mart. - todas não-ornitófilas. Beija-flores desta espécie exploraram flores com corolas curtas, médias e longas e visitaram ilegitimamente as flores de $P$. cf. melioides e algumas flores de $H$. velutina, utilizando perfurações já existentes na corola.

Os indivíduos de C. lucidus avistados entre as sessões de visitas permaneciam empoleirados próximos às plantas floridas, defendendo território com interações agonísticas, não tendo sido observados machos e fêmeas partilhando um mesmo território.

Foram observadas 37 interações agonísticas, das quais 21 foram interespecíficas (Tab. III). Chlorostilbon lucidus foi a espécie com maior número de agonismos registrados: 28 registros, doze dos quais interespecíficos (nove destes indivíduos machos e

Tabela I. Espécies de beija-flores registradas entre outubro de 2005 e agosto de 2007, em área de caatinga arbórea na Chapada Diamantina, município de Mucugê, Bahia. Padrão sazonal: (RE) residente, (NR) não-residente.

\begin{tabular}{|c|c|c|c|c|c|c|c|c|c|c|c|c|c|}
\hline \multirow{3}{*}{ Subfamília / Espécie } & \multicolumn{12}{|c|}{ Ano } & \multirow{3}{*}{ Padrão sazonal } \\
\hline & \multicolumn{2}{|c|}{2005} & \multicolumn{6}{|c|}{2006} & \multicolumn{4}{|c|}{2007} & \\
\hline & Out & Dez & Fev & Abr & Jun & Ago & Out & $\mathrm{Dez}$ & Fev & Abr & Jun & Ago & \\
\hline \multicolumn{14}{|l|}{ Trochilinae } \\
\hline Anopetia gounellei (Boucard, 1891) & & $x$ & & $x$ & $x$ & $x$ & $x$ & $x$ & $x$ & $x$ & $x$ & $x$ & RE \\
\hline Phaethornis pretrei (Lesson \& Delattre, 1839) & $x$ & $x$ & $x$ & $x$ & $x$ & $\mathrm{X}$ & $\mathrm{x}$ & $x$ & $x$ & $x$ & $\mathrm{X}$ & $x$ & RE \\
\hline \multicolumn{14}{|l|}{ Phaethornithinae } \\
\hline Eupetomena macroura (Gmelin, 1788) & & & & & & $\mathrm{X}$ & $x$ & & $x$ & & & & NR \\
\hline Chrysolampis mosquitus (Linnaeus, 1758) & & $x$ & $x$ & $x$ & & $x$ & $x$ & & $x$ & $x$ & $x$ & $x$ & RE \\
\hline Colibri serrirostris (Vieillot, 1816) & & & & & & & & & $\mathrm{x}$ & & & & NR \\
\hline Chlorostilbon lucidus (Shaw, 1812) & $x$ & $x$ & $x$ & $x$ & $x$ & $\mathrm{X}$ & $\mathrm{X}$ & $\mathrm{x}$ & $x$ & $x$ & $\mathrm{X}$ & $x$ & RE \\
\hline Amazilia lactea (Lesson, 1832) & & $x$ & $x$ & $x$ & $x$ & $\mathrm{X}$ & $x$ & & $x$ & $x$ & $x$ & $x$ & RE \\
\hline
\end{tabular}


Tabela II. Espécies de plantas visitadas por beija-flores em uma área de caatinga arbórea da Chapada Diamantina, no município de Mucugê, Bahia, de outubro de 2005 a agosto de 2007. Tipo de flor: (TB) tubo, (PT) pseudo-tubo, (GO) goela, (CM) campânula, (FU) funil, (PI) pincel, (DI) disco, (CP) capítulo, (ST) estandarte. Cor da flor: (VM) vermelha, (AM) amarela, (BR) branca, (LI) lilás, (LR) laranja, (RS) rosa, (AZ) azul, (MR) marrom. Porte: (ABV) arbustivo, (HER) herbáceo, (ARB) arbóreo/subarbóreo, (LIA) liana. Síndrome - Síndrome de polinização: (OR) ornitofilia, $(E M)$ entomofilia, $(Q U)$ quiropterofilia. Comprimento do tubo: média do comprimento da corola \pm desvio padrão; entre parênteses, número de flores amostradas. Concentração de néctar: Concentração média do néctar \pm desvio padrão; entre parênteses, número de flores amostradas. Beija flores visitantes: (ANO) Anopetia gounellei, (PHA) Phaethornis pretrei, (EUP) Eupetomena macroura, (CHR) Chrysolampis mosquitus, (COL) Colibri serrirostris, (CHL) Chlorostilbon lucidus, (AMA) Amazilia lactea, (-) sem registro.

\begin{tabular}{|c|c|c|c|c|c|c|c|}
\hline Família / Espécie & $\begin{array}{l}\text { Tipo } \\
\text { de flor }\end{array}$ & Cor & Porte & Síndrome & $\begin{array}{l}\text { Comprimento } \\
\text { do tubo }(\mathrm{mm})\end{array}$ & $\begin{array}{l}\text { Concentração } \\
\text { de néctar (\%) }\end{array}$ & Beija-flores visitantes \\
\hline \multicolumn{8}{|l|}{ Acanthaceae } \\
\hline Anisacanthus brasiliensis & GO & VM & $A B V$ & OR & $23,6 \pm 1,5(10)$ & $31,0 \pm 0(10)$ & $\mathrm{CHL}, \mathrm{AMA}, \mathrm{PHA}, \mathrm{ANO}$ \\
\hline Ruellia sp. & $\mathrm{CM}$ & $\mathrm{LI}$ & HER & EN & $48,0 \pm 1,4(10)$ & $28,2 \pm 3,1(10)$ & $\mathrm{PHA}, \mathrm{CHR}, \mathrm{CHL}$ \\
\hline \multicolumn{8}{|l|}{ Alstroemeriaceae } \\
\hline Alstroemeria rupestris & PT & LR/ VM & HER & OR & $40,1 \pm 1,6(10)$ & $33,0 \pm 0(6)$ & PHA \\
\hline \multicolumn{8}{|l|}{ Apocynaceae } \\
\hline Mandevilla scabra & $\mathrm{CM}$ & AM/VM & LIA & EN & $43,1 \pm 0,2(3)$ & - & PHA \\
\hline Prestonia coalita & TB & $\mathrm{AM}$ & LIA & EN & $2,5 \pm 0,2(10)$ & $36,3 \pm 5,3(5)$ & $\mathrm{AMA}, \mathrm{CHL}, \mathrm{CHR}, \mathrm{ANO}$ \\
\hline \multicolumn{8}{|l|}{ Asteraceae } \\
\hline Dasyphyllum candolleanum & $\mathrm{CP}$ & $\mathrm{BR}$ & $A B V$ & EN & - & - & $\mathrm{CHL}$ \\
\hline \multicolumn{8}{|l|}{ Bignoniaceae } \\
\hline Setilobus simplicifolius & $\mathrm{CM}$ & $\mathrm{AM}$ & $A B V$ & EN & $45,1 \pm 0,8(10)$ & - & $\begin{array}{l}\text { AMA, ANO, CHR, CHL, PHA, } \\
\text { COL, EUP }\end{array}$ \\
\hline Pyrostegia venusta & TB & LR & LIA & OR & $55,4 \pm 2,8(10)$ & $24,3 \pm 1,5(6)$ & PHA, EUP, ANO, AMA, CHR \\
\hline Piriadacus erubescens & TB & VM & $A B V$ & OR & $47,5 \pm 1,9(6)$ & $22,2 \pm 0,5(4)$ & ANO, AMA, PHA, CHR \\
\hline Pleonotoma cf. melioides & GO & $\mathrm{BR} / \mathrm{RS} / \mathrm{AM}$ & LIA & EN & $47,2 \pm 0,6(5)$ & $39,8 \pm 0,2(4)$ & $\mathrm{CHL}$ \\
\hline Bignoniaceae sp. & GO & RS & LIA & EN & $49,1 \pm 3,2(10)$ & $26,2 \pm 4,9(8)$ & CHR, AMA, ANO \\
\hline \multicolumn{8}{|l|}{ Boraginaceae } \\
\hline Cordia superba & $\mathrm{FN}$ & $B R$ & $A B V$ & EN & $32,2 \pm 1,8(10)$ & $26,4 \pm 0,5(10)$ & PHA, AMA, CHL \\
\hline \multicolumn{8}{|l|}{ Bromeliaceae } \\
\hline Orthophytum lemei & TB & $\mathrm{AM} / \mathrm{VM}$ & HER & OR & $14,2 \pm 1,7(6)$ & $25,5 \pm 0(6)$ & $\mathrm{CHL}, \mathrm{PHA}$ \\
\hline \multicolumn{8}{|l|}{ Campanulaceae } \\
\hline Centropogon cornutus & $\mathrm{GO}$ & VM & $A B V$ & OR & $47,9 \pm 0,5(6)$ & $22,0 \pm 0(3)$ & PHA \\
\hline \multicolumn{8}{|l|}{ Convolvulaceae } \\
\hline Jacquemontia sp. 1 & $\mathrm{FN}$ & $A Z$ & HER & EN & $19,8 \pm 1,1(5)$ & - & AMA \\
\hline Jacquemontia sp. 2 & $\mathrm{FN}$ & $\mathrm{BR}$ & LIA & EN & $18,2 \pm 1,0(10)$ & - & $\mathrm{AMA}, \mathrm{CHL}$ \\
\hline \multicolumn{8}{|l|}{ Fabaceae } \\
\hline Bauhinia longifolia & $\mathrm{PI}$ & MR/VM & ARB & QU/OR & - & $23,0 \pm 0,5(5)$ & $\mathrm{CHL}, \mathrm{PHA}$ \\
\hline Chaetocalyx subulatus & ST & AM & LIA & EN & - & - & $\mathrm{CHL}$ \\
\hline Periandra coccinea & ST & VM & $A B V$ & OR & - & - & $\mathrm{PHA}, \mathrm{CHL}$ \\
\hline \multicolumn{8}{|l|}{ Lamiaceae } \\
\hline Hyptis leptostachys & TB & $A Z$ & $A B V$ & EN & $7,5 \pm 0,3(10)$ & $31,5 \pm 0(6)$ & $\mathrm{CHL}, \mathrm{AMA}$ \\
\hline \multicolumn{8}{|l|}{ Lythraceae } \\
\hline Lafoensia glyptocarpa & $\mathrm{PI}$ & $\mathrm{BR}$ & $A B V$ & QU & - & - & $\mathrm{CHL}$ \\
\hline & & & & & & & $\mathrm{Co}$ \\
\hline
\end{tabular}


Tabela II.Continuação.

\begin{tabular}{|c|c|c|c|c|c|c|c|}
\hline Família / Espécie & $\begin{array}{l}\text { Tipo } \\
\text { de flor }\end{array}$ & Cor & Porte & Síndrome & $\begin{array}{l}\text { Comprimento } \\
\text { do tubo }(\mathrm{mm})\end{array}$ & $\begin{array}{l}\text { Concentração } \\
\text { de néctar (\%) }\end{array}$ & Beija-flores visitantes \\
\hline \multicolumn{8}{|l|}{ Malvaceae } \\
\hline Pavonia sp. & PT & VM & $A B V$ & OR & $20,0 \pm 6,3(10)$ & $20,3 \pm 2,0(8)$ & AMA, ANO, PHA, CHL, CHR \\
\hline \multicolumn{8}{|l|}{ Passifloraceae } \\
\hline Passiflora edmundoi & $\mathrm{DI}$ & VM & LIA & OR & - & - & $\mathrm{PHA}, \mathrm{CHL}$ \\
\hline \multicolumn{8}{|l|}{ Rubiaceae } \\
\hline Augusta longifolia & TB & VM & $A B V$ & OR & $56,0 \pm 4,6(10)$ & $21,5 \pm 1,3(10)$ & PHA \\
\hline \multicolumn{8}{|l|}{ Sapindaceae } \\
\hline Serjania coradinii & $\mathrm{DI}$ & BR & LIA & EN & - & - & $\mathrm{CHL}, \mathrm{ANO}, \mathrm{PHA}$ \\
\hline \multicolumn{8}{|l|}{ Sterculiaceae } \\
\hline Helicteres eichleri & TB & $\mathrm{AM} / \mathrm{VM}$ & $A B V$ & OR & $21,9 \pm 0,7(10)$ & $21,2 \pm 2,0(10)$ & CHR, AMA, PHA, CHL \\
\hline Helicteres velutina & TB & VM & $A B V$ & OR & $20,4 \pm 1,4(10)$ & $22,9 \pm 1,7(10)$ & $\begin{array}{l}\mathrm{CHL}, \mathrm{AMA}, \mathrm{PHA}, \mathrm{CHR}, \mathrm{ANO}, \\
\mathrm{COL}\end{array}$ \\
\hline \multicolumn{8}{|l|}{ Verbenaceae } \\
\hline Lantana camara & $\mathrm{TB}$ & $\mathrm{AZ} / \mathrm{VM} / \mathrm{LR}$ & $A B V$ & EN & $5,8 \pm 0,5(10)$ & - & $\mathrm{CHL}$ \\
\hline Stachitarpheta quadrangula & TB & $A Z$ & HER & EN & $19,0 \pm 0,6(6)$ & - & $\mathrm{CH}$ \\
\hline
\end{tabular}

Tabela III. Matriz das interações agressivas registradas entre beija-flores (Aves: Trochilidae) em uma área de caatinga, no município de Mucugê, Chapada Diamantina, Bahia, no período de outubro de 2005 a agosto de 2007. Para cada espécie foi feita a somatória dos eixos horizontal (número de vezes que cada espécie atacou) e vertical (número de vezes que a espécie foi atacada), onde $\Sigma^{1}=$ somatória das interações interespecíficas e $\Sigma^{2}=$ somatória total. Os valores em negrito correspondem às interações intra-específicas. (ANO) Anopetia gounellei, (PHA) Phaethornis pretrei, (EUP) Eupetomena macroura, (CHR) Chrysolampis mosquitus, (COL) Colibri serrirostris, (CHL) Chlorostilbon lucidus, (AMA) Amazilia lactea.

\begin{tabular}{|c|c|c|c|c|c|c|c|c|c|}
\hline \multirow{2}{*}{ Espécies dominantes } & \multicolumn{9}{|c|}{ Espécies subordinadas } \\
\hline & ANO & $\mathrm{PHA}$ & AMA & $\mathrm{CHL}$ & $\mathrm{CHR}$ & $\mathrm{COL}$ & EUP & $\Sigma^{1}$ & $\Sigma$ \\
\hline ANO & - & - & - & - & - & - & - & 0 & 0 \\
\hline PHA & - & 4 & - & 1 & - & - & - & 0 & 5 \\
\hline AMA & - & - & 3 & 6 & - & - & - & 6 & 9 \\
\hline $\mathrm{CHL}$ & - & - & 4 & 12 & 2 & - & - & 7 & 18 \\
\hline $\mathrm{CHR}$ & - & - & - & 3 & 2 & - & - & 3 & 5 \\
\hline $\mathrm{COL}$ & - & - & - & - & - & - & - & 0 & 0 \\
\hline EUP & - & - & - & - & - & - & - & 0 & 0 \\
\hline$\Sigma^{1}$ & 0 & 0 & 4 & 10 & 2 & 0 & 0 & 16 & - \\
\hline$\Sigma^{2}$ & 0 & 4 & 7 & 22 & 4 & 0 & 0 & - & 37 \\
\hline
\end{tabular}

fêmeas). Também ocorreram agonismos interespecíficos entre P. pretrei, A. lactea e C. mosquitus - neste último, os dois eventos observados foram entre machos.

As 29 espécies de plantas visitadas por beija-flores, na área amostrada, pertencem a 18 famílias (Tab. I), sendo que Bignoniaceae contribuiu com o maior número de espécies (cinco), seguida por Fabaceae (três espécies). As famílias
Acanthaceae, Apocynaceae, Convolvulaceae, Sterculiaceae e Verbenaceae contaram com duas espécies registradas e as 11 famílias restantes, com uma espécie.

Das espécies que foram exploradas pelos beija-flores, 16 foram categorizadas não ornitófilas (15 entomófilas e uma quiropterófila), 12 ornitófilas e uma (Bauhinia longifolia D. Dietr.) de síndrome mista (quiropterófila/ornitófila) (Tab. II). 
Quanto ao porte, 14 espécies foram arbustos, nove lianas, cinco de hábito herbáceo e apenas uma de arbóreo-arbustivo ( $B$. longifolia) (Tab. II). As espécies visitadas têm, em geral, flores tubulosas (12 espécies), com corolas longas (11 espécies) e com coloração de suas partes ou anexos florais vermelhos (11 espécies) (Tab. II).

As concentrações do néctar das espécies visitadas variaram de $20,3 \%$ (Pavonia sp.) a 39, 8 \% (P. cf. melioides) (Tab. II), e em 12 espécies não foi detectada a presença de néctar ou seu volume era pequeno demais para a análise de sua concentração.

A maioria das espécies de plantas registradas ocorreu ao longo de toda a área estudada, como, por exemplo, Ruellia sp., Prestonia coalita (Vell.) Woodson, D. candolleanum, C. subulatus, Hyptis leptostachys Epling subs. caatingae Harley, Pavonia sp., $H$. velutina, Serjania coradinii Ferrucci \& Somner. Algumas ocorreram de forma mais agregada, formando manchas, como Anisacanthus brasiliensis Lindau, S. simplicifolius, P. cf. melioides, Bignoniaceae sp. e L. camara. Outras ocorreram isoladas, como A. rupestris, Centropogon cornutus (Linnaeus) Druce (restrita às áreas úmidas, com terreno encharcado), A. longifolia (espécie reófita) e Orthophytum lemei Pereira \& Penna (bromélia rupícola).

A correlação entre o comprimento dos bicos dos beijaflores e o do tubo das corolas das flores que forrageavam foi positiva, porém não significativa $(\mathrm{r}=0,13 ; \mathrm{p}>0,05 ; \mathrm{n}=53)$.

Dezessete espécies tiveram um padrão fenológico de floração coerente com o tipo breve e anual, cinco espécies como do tipo longo e contínuo, cinco de do tipo intermediário, sendo três de floração contínua e duas anuais; uma espécie encaixase no tipo longo e anual e outra como breve e supra-anual (Tab. IV). Dezenove espécies floresceram tanto na estação seca quanto na chuvosa, seis floresceram exclusivamente na estação seca e quatro apenas na chuvosa (Tab. IV).

O agrupamento das espécies de beija-flor em função de sua similaridade de uso de plantas revelou dois grupos, sendo um formado apenas por $P$. pretrei e $C$. lucidus e o outro pelas demais espécies.

\section{DISCUSSÃO}

O total de espécies de beija-flores registrado neste estudo representa aproximadamente $43 \%$ da troquilofauna registrada em todo o bioma Caatinga, da qual constam 28 espécies (Silva et al. 2003). Os registros de P. pretrei, A. lactea, C. mosquitus e $C$. serrirostris, efetuados no presente estudo, ampliaram a riqueza da troquilofauna em área de caatinga da Chapada Diamantina para 12 espécies. Estudos realizados previamente em áreas de caatinga dessa região (PARRINI et al. 1999, MACHADO 2005) haviam detectado oito espécies de beija-flores, concentradas em áreas mais úmidas, como a Serra do Bastião. Das espécies de troquilídeos reportadas por esses autores, apenas $A$. gounellei (endêmica de vegetação de caatinga), E. macroura e C. lucidus foram registradas no presente estudo.

Phaethornis pretrei e C. lucidus foram consideradas as únicas espécies residentes em uma área de campo rupestre na
Chapada Diamantina (MACHADo et al. 2007). No presente estudo, além destas duas espécies, outras três (A. gounellei, C. mosquitus e $A$. lactea) ocorreram praticamente durante todo o período estudado, indicando serem também residentes em área de caatinga arbórea.

Em uma área de campo rupestre da Chapada Diamantina, a cerca de $40 \mathrm{~km}$ da área de caatinga amostrada no presente estudo, Machado et al. (2007) notaram a ausência de A. lactea, C. serrirostris e C. mosquitus no período mais seco. Assim, aventase a possibilidade de que áreas florestadas, como a caatinga arbórea da Serra do Bastião, sejam destinos para as populações de beija-flores de áreas campestres que têm uma menor capacidade para manter as populações destas aves nos períodos mais secos. O acompanhamento de indivíduos anilhados pode testar esta hipótese.

Colibri serrirostris se comportou, no presente estudo, como espécie não-residente, devido a sua ocorrência ocasional. Tratase de uma espécie campestre (SiLva et al. 2003) incomum em ambientes florestais. Eupetomena macroura também tem um padrão de ocorrência que pode ser interpretado como nãoresidente, sendo classificada ocasional, pois sua ocorrência na área foi irregular e pouco freqüente.

Phaethornis pretrei e A. gounellei apresentaram estratégia de forrageio típica de Phaethornitinae (Feinsinger \& Colwell 1978), forrageando em rotas de captura ("traplining"), ou seja, deslocando-se por uma grande área em busca de recursos. Esta estratégia foi reconhecida devido aos intervalos de visitação bastante espaçados, de 60 a 90 minutos (ou mais), e também pela ausência de comportamentos de defesa de territórios de exploração, junto às manchas de recursos. Os indivíduos dessas espécies também foram mais seletivos quanto aos recursos explorados, visitando predominantemente flores ornitófilas mais especializadas, com corolas vermelhas, de tubo longo e encurvados, de plantas isoladas (como A. longifolia e A. rupestris, visitadas exclusivamente por $P$. pretrei).

Por outro lado, os troquilíneos amostrados no presente estudo foram considerados tipicamente territorialistas, defendendo áreas restritas de forrageamento, e generalistas, explorando um maior número de espécies não especializadas (não-ornitófilas). Chlorostilbon lucidus e A. lactea estiveram envolvidos na maioria das interações agonísticas observadas na área de caatinga, como também foi observado em área de campo rupestre da Chapada Diamantina (MACHADo et al. 2007). Ambas as espécies (e tanto machos quanto fêmeas) possuem tamanho e forma de bicos similares (GranTSAU 1988), o que pode levar a uma sobreposição do uso recursos (tamanho e tipo de flores). Os agonismos podem separar territórios de forrageamento, resultando em uma partilha de recursos e diminuindo, deste modo, a competição intra e interespecífica. Essa distinção na utilização de recursos pode ser detectada na análise de agrupamento (Fig. 1), que separou C. lucidus em um grupo distinto de $A$. lactea.

Indivíduos de $P$. pretrei e C. lucidus visitaram a maior 
Tabela IV. Espécies de plantas e de seus beija-flores visitantes (Aves: Trochilidae) e seu padrão de floração em uma área de caatinga em Mucugê, Chapada Diamantina, Bahia, no período de outubro de 2005 a agosto de 2006. (NI) Número de indivíduos monitorados, (NV) número total de sessões de visitas de beija-flores registrado, $(\mathrm{NH})$ número total de horas de observação, (FT) freqüência total de visitas de beija-flores (sessões de visitas por indivíduo/hora de observação). Freqüência relativa de visitas (\%): (ANO) Anopetia gounellei, (PHA) Phaethornis pretrei, (EUP) Eupetomena macroura, (CHR) Chrysolampis mosquitus, (COL) Colibri serrirostris, (CHL) Chlorostilbon lucidus, (AMA) Amazilia lactea. Padrão fenológico de floração: (BR) breve, (IN) intermediário, (LO) longo, (CO) contínuo, (SB) subanual, (AN) anual, (SP) supra-anual. Período de floração: (EC) estação chuvosa, (ES) estação seca.

\begin{tabular}{|c|c|c|c|c|c|c|c|c|c|c|c|c|c|c|}
\hline \multirow{2}{*}{ Família /Espécie } & \multirow{2}{*}{$\mathrm{NI}$} & \multirow{2}{*}{ NV } & \multirow{2}{*}{$\mathrm{NH}$} & \multirow{2}{*}{ FT } & \multicolumn{7}{|c|}{ Freqüência relativa de visitas (\%) } & \multirow{2}{*}{$\begin{array}{l}\text { Padrão de } \\
\text { floração }\end{array}$} & \multicolumn{2}{|c|}{$\begin{array}{l}\text { Período de } \\
\text { floração }\end{array}$} \\
\hline & & & & & ANO & PHA & EUP & $\mathrm{CHR}$ & $\mathrm{COL}$ & $\mathrm{CHL}$ & AMA & & $\mathrm{EC}$ & ES \\
\hline \multicolumn{15}{|l|}{ Acanthaceae } \\
\hline Anisacanthus brasiliensis & 38 & 565 & 418 & 1,4 & 1,8 & 22,5 & - & - & - & 38,6 & 37,2 & $\mathrm{CO}$ & $x$ & $x$ \\
\hline Ruellia sp. & 15 & 123 & 164 & 0,8 & - & 67,5 & - & 24,4 & - & 8,1 & - & $\mathrm{CO}$ & $x$ & $x$ \\
\hline Alstroemeriaceae & & & & & - & 100 & - & - & - & - & - & & & \\
\hline Alstroemeria rupestris & 7 & 88 & 78 & 1,1 & - & 100 & - & - & - & - & - & $\mathrm{BR}, \mathrm{AN}$ & & $x$ \\
\hline \multicolumn{15}{|l|}{ Apocynaceae } \\
\hline Mandevilla scabra & 3 & 6 & 22 & 0,8 & - & 100 & - & - & - & - & - & $\mathrm{BR}, \mathrm{AN}$ & & $x$ \\
\hline Prestonia coalita & 18 & 157 & 200 & 0,8 & 0,6 & - & - & 1,9 & - & 42,0 & 55,4 & BR, AN & $x$ & $x$ \\
\hline \multicolumn{15}{|l|}{ Asteraceae } \\
\hline Dasyphyllum candolleanum & 3 & 4 & 30 & 0,1 & - & - & - & - & - & 100 & - & BR, AN & $x$ & $x$ \\
\hline \multicolumn{15}{|l|}{ Bignoniaceae } \\
\hline Setilobus simplicifolius & 13 & 97 & 130 & 0,7 & 21,6 & 10,3 & 2,0 & 20,6 & 7,2 & 11,3 & 26,8 & $\mathrm{BR}, \mathrm{AN}$ & $x$ & $x$ \\
\hline Pyrostegia venusta & 6 & 40 & 92 & 0,4 & 10,0 & 50,0 & 30,0 & 2,5 & - & - & 7,5 & BR, AN & & $x$ \\
\hline Piriadacus erubescens & 2 & 17 & 20 & 0,8 & 35,3 & 23,5 & - & 5,9 & - & - & 35,3 & $\mathrm{BR}, \mathrm{AN}$ & $x$ & \\
\hline Pleonotoma cf. melioides & 1 & 4 & 10 & 0,4 & - & - & - & - & - & 100 & - & $\mathrm{BR}, \mathrm{AN}$ & $x$ & \\
\hline Bignoniaceae sp. & 9 & 207 & 111 & 1,9 & 1,9 & - & - & 55,1 & - & - & 43,5 & BR, AN & $x$ & \\
\hline \multicolumn{15}{|l|}{ Boraginaceae } \\
\hline Cordia superba & 5 & 11 & 31 & 0,3 & - & 63,6 & - & - & - & 18,2 & 18,2 & BR, AN & $x$ & $x$ \\
\hline \multicolumn{15}{|l|}{ Bromeliaceae } \\
\hline Orthophytum lemei & 7 & 62 & 70 & 0,9 & - & 17,7 & - & - & - & 82,2 & & BR, AN & $x$ & $x$ \\
\hline \multicolumn{15}{|l|}{ Campanulaceae } \\
\hline Centropogon cornutus & 18 & 110 & 131 & 0,8 & - & 100 & - & - & - & - & - & $B R, S B$ & $x$ & $x$ \\
\hline \multicolumn{15}{|l|}{ Convolvulaceae } \\
\hline Jacquemontia sp. 1 & 4 & 19 & 45 & 0,4 & - & - & - & - & - & 20,0 & 80,0 & $\mathrm{BR}, \mathrm{AN}$ & $x$ & $x$ \\
\hline Jacquemontia sp. 2 & 1 & 5 & 10 & 0,5 & - & - & - & - & - & - & 100 & BR, AN & $x$ & $x$ \\
\hline \multicolumn{15}{|l|}{ Fabaceae } \\
\hline Bauhinia longifolia & 3 & 42 & 46 & 0,9 & - & 52,4 & - & - & - & 47,6 & - & $\mathrm{CO}$ & $x$ & $x$ \\
\hline Chaetocalyx subulatus & 9 & 18 & 102 & 0,2 & - & - & - & - & - & 100 & - & BR, AN & & $x$ \\
\hline Periandra coccinea & 13 & 31 & 121 & 0,2 & - & 64,5 & - & - & - & 35,5 & - & $\mathrm{CO}$ & $x$ & $x$ \\
\hline \multicolumn{15}{|l|}{ Lamiaceae } \\
\hline Hyptis leptostachys & 36 & 137 & 400 & 0,3 & - & - & - & - & - & 90,5 & 9,5 & IN, AN & $x$ & $x$ \\
\hline \multicolumn{15}{|l|}{ Lythraceae } \\
\hline Lafoensia glyptocarpa & 2 & 11 & 24 & 0,4 & - & - & - & - & - & 100 & - & $\mathrm{BR}, \mathrm{AN}$ & & $x$ \\
\hline
\end{tabular}


Tabela IV. Continuação.

\begin{tabular}{|c|c|c|c|c|c|c|c|c|c|c|c|c|c|c|}
\hline \multirow{2}{*}{ Família /Espécie } & \multirow{2}{*}{$\mathrm{NI}$} & \multirow{2}{*}{ NV } & \multirow{2}{*}{$\mathrm{NH}$} & \multirow[t]{2}{*}{ FT } & \multicolumn{7}{|c|}{ Freqüência relativa de visitas (\%) } & \multirow{2}{*}{$\begin{array}{l}\text { Padrão de } \\
\text { floração }\end{array}$} & \multicolumn{2}{|c|}{$\begin{array}{l}\text { Período de } \\
\text { floração }\end{array}$} \\
\hline & & & & & ANO & PHA & EUP & $\mathrm{CHR}$ & $\mathrm{COL}$ & $\mathrm{CHL}$ & AMA & & $\mathrm{EC}$ & ES \\
\hline \multicolumn{15}{|l|}{ Malvaceae } \\
\hline Pavonia sp. & 18 & 299 & 177 & 1,7 & 26,0 & 7,2 & - & 0,3 & - & 3,4 & 65,4 & $\mathrm{CO}$ & $x$ & $x$ \\
\hline \multicolumn{15}{|l|}{ Passifloraceae } \\
\hline Passiflora edmundoi & 7 & 21 & 71 & 0,3 & - & 90,5 & - & - & - & 9,5 & - & IN, AN & $x$ & $x$ \\
\hline \multicolumn{15}{|l|}{ Rubiaceae } \\
\hline Augusta longifolia & 5 & 27 & 53 & 0,5 & - & 100 & - & - & - & - & - & $\mathrm{BR}, \mathrm{AN}$ & $x$ & \\
\hline \multicolumn{15}{|l|}{ Sapindaceae } \\
\hline Serjania coradinii & 30 & 71 & 336 & 0,2 & 5,7 & 4,2 & - & - & - & 90,1 & - & $\mathrm{LO}, \mathrm{AN}$ & $x$ & $x$ \\
\hline \multicolumn{15}{|l|}{ Sterculiaceae } \\
\hline Helicteres eichleri & 4 & 19 & 43 & 0,4 & - & 15,8 & - & 47,4 & - & 15,8 & 21,0 & $\mathrm{CO}$ & $x$ & $x$ \\
\hline Helicteres velutina & 38 & 352 & 407 & 0,9 & 4,0 & 15,9 & - & 6,0 & 0,6 & 52,8 & 17,9 & $\mathrm{CO}$ & $x$ & $x$ \\
\hline \multicolumn{15}{|l|}{ Verbenaceae } \\
\hline Lantana camara & 5 & 7 & 50 & 0,1 & - & - & - & 100 & - & - & - & $\mathrm{CO}$ & $\mathrm{X}$ & $x$ \\
\hline Stachitarpheta quadrangula & 2 & 2 & 20 & 0,1 & - & - & - & 100 & - & - & - & $\mathrm{BR}, \mathrm{AN}$ & & $x$ \\
\hline
\end{tabular}

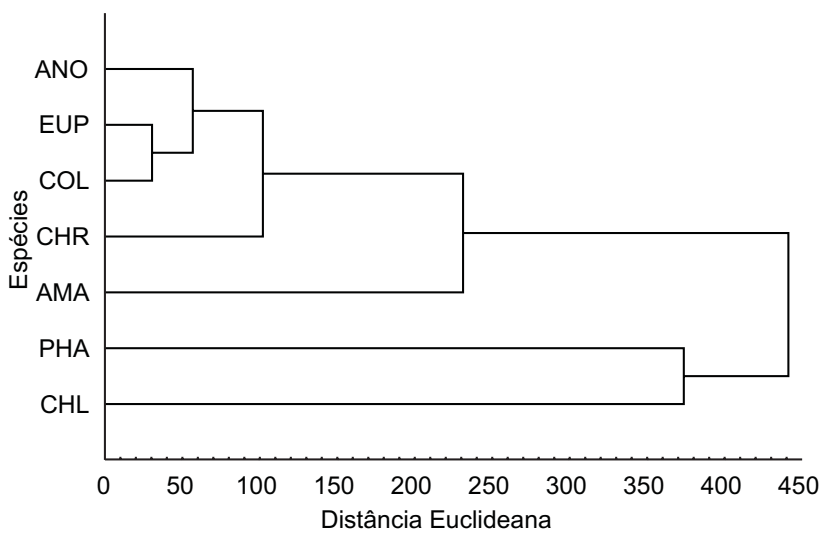

Figura 1. Análise de agrupamento hierárquico das espécies de beijaflores de uma área de caatinga arbórea no município de Mucugê, Chapada Diamantina, Bahia, pela similaridade de espécies de plantas que visitam, considerando as suas freqüências de visitas. (ANO) Anopetia gounellei, (PHA) Phaethornis pretrei, (EUP) Eupetomena macroura, (CHR) Chrysolampis mosquitus, (COL) Colibri serrirostris, (CHL) Chlorostilbon lucidus, (AMA) Amazilia lactea.

diversidade de espécies de plantas, muitas delas comuns a ambos, colocando-os em um mesmo conjunto, quando agrupados pela similaridade na utilização de recursos. Essa capacidade destes dois troquilídeos explorarem uma maior diversidade de recursos também ocorre em ambiente de campo rupestre (MACHADo et al. 2007). No presente estudo, indivíduos de $P$. pretrei também utilizaram um maior número de espécies com flores mais especializadas, ornitófilas de corolas longas e tubulosas, enquanto que os de C. lucidus, visitaram um conjunto mais variado de tipos florais, com cores e tamanhos mais diversos.

Por isso e por serem espécies residentes e bastante freqüentes, pode-se classificá-las como polinizadores principais, sendo as demais espécies residentes polinizadoras secundárias, conforme FeIsinger \& Colwell (1978).

Phaethornis pretrei pode ser considerada como espécie organizadora desta guilda de polinizadores (cf. FeIsINGer \& Colwell 1978) uma vez que explorou legitimamente um maior número de espécies ornitófilas, mais especializadas. Fetornitíneos são reconhecidos como organizadores deste tipo de comunidades em outros estudos (SAzIma et al. 1996, BuZATo et al. 2000), embora Leal et al. (2006) tenham encontrado C. lucidus (Trochilinae) exercendo este papel em uma comunidade de plantas ornitófilas em outra área de caatinga, em Pernambuco. Estes autores consideraram que, devido à escassez de plantas com requisitos energéticos e características florais adequadas, aquela comunidade vegetal não pode sustentar um fetornitíneo como espécie organizadora.

Os beija-flores utilizaram flores de 29 espécies de plantas, riqueza semelhante à encontrada em diversas áreas de florestas na Mata Atlântica (BuzATo et al. 2000) e em campo rupestre, também na Chapada Diamantina (MACHado et al. 2007).

Em outros ambientes tropicais é registrada uma maior riqueza de espécies de plantas utilizadas por troquilídeos floridas na estação chuvosa (Fischer \& Araujo 1995, Buzato et al. 2000, Machado \& Semir 2006); entretanto, LeAL et al. (2006) reportam 
a ocorrência, na área de caatinga que estudaram, de uma maior floração de espécies ornitófilas, tanto em riqueza quanto intensidade, na estação seca. Assim como também observado em área de campo rupestre da Chapada Diamantina (MACHADO et al. 2007), a maioria das espécies aqui estudadas floresceu tanto na estação seca como chuvosa.

Todas as visitas feitas pelos beija-flores foram legítimas, à exceção das realizadas em duas espécies de bignoniáceas nãoornitófilas. O comportamento dos beija-flores de realizar visitas legítimas, tanto em espécies ornitófilas quanto não-ornitófilas, observado neste estudo, pode ocasionar o contato com as partes férteis das flores, fazendo com que estas aves atuem como vetores de pólen. Alguns autores (Brown \& Bowers 1985, Fenster 1991) consideram que o acesso ao néctar em flores com corola longa seja restrito aos beija-flores de bico longo, impedindo que visitantes de bico curto alcancem o néctar contido no fundo da corola. A priori, no presente estudo, essa idéia parece ser pertinente quando se analisa plantas com flores mais especializadas, como A. longifolia, C. cornutus e A. rupestris, todas com corolas longas e vermelhas, que são visitadas exclusivamente por $P$. pretrei, espécie com bico longo.

No entanto, ao analisar a comunidade como um todo, observa-se que, assim como também em diversos outros estudos (Fischer \& Araujo 1995, Sazima et al. 1996, Buzato et al. 2000, MACHADO \& SEMIR 2006, MACHADo et al. 2007), não há correlação significativa entre as variáveis profundidade da corola e comprimento do bico, uma vez que beija-flores de bico longo também visitam espécies de corola curta (p.e. $P$. pretrei e $S$. coradinii) e as espécies com bico curto também exploram flores de corola grande - neste caso as do tipo campânula e goela, que não apresentam tubos estreitos.

Este resultado corrobora o encontrado por Temeles \& LinHART (2002), os quais discutiram a relação sobre o tamanho de bicos de beija-flores com a profundidade das corolas das flores que exploram e concluíram que, na maioria dos casos, sobretudo em ambientes tropicais, a coevolução destes dois grupos tende a ser mais difusa.

Estudos para determinação precisa dos padrões fenológicos de floração requerem acompanhamento mensal das comunidades vegetais estudadas (Newstrom et al. 1994); entretanto, mesmo com as amostragens bimestrais efetuadas no presente estudo, pode-se inferir que a comunidade de plantas que são utilizadas por beija-flores, nesta área de caatinga, apresenta o padrão fenológico de floração seqüencial, com sobreposição dos períodos de floração das diversas espécies, ocorrendo continuidade de recursos alimentares disponíveis.

Este padrão fenológico de floração, registrado também em outras comunidades (Fischer \& Araujo 1995, Buzato et al. 2000, Machado \& Semir 2006, MACHADO et al. 2007), propicia à troquilofauna recursos alimentares ao longo de todo o ano, viabilizando a permanência de diversas espécies residentes. Para as plantas, segundo Poulin et al. (1992), há a conservação de seus visitantes florais na área, assegurando que os primeiros indivíduos a florescer poderão contar com a presença de seus polinizadores, garantindo seu sucesso reprodutivo.

A importância das diferentes espécies vegetais na dieta dos beija-flores na área da caatinga arbórea pode ser avaliada considerando a freqüência com que são visitadas por estas aves. Espécies ornitófilas tenderam a ter uma maior média de freqüência de visitação. Dentre estas, as mais especializadas, com corola vermelha e tubulosa, como A. brasiliensis, A. rupestris, Piriadacus erubescens (DC.) Pichon, C. cornutus e Pavonia sp., apresentaram as maiores freqüência de visitas pelos beija-flores.

Estudos sobre uso de néctar de plantas ornitófilas e nãoornitófilas como recursos para beija-flores têm evidenciado que estas últimas podem representar significativa porção em sua dieta, havendo influência de fatores como a disponibilidade de recursos e competição (SNOw \& SNOw 1986, Arizmendi \& Ornelas 1990, Sazima et al. 1996, Leal et al. 2006, Machado \& SEMIR 2006, Machado et al. 2007).

$\mathrm{Na}$ comunidade de plantas visitadas por beija-flores em ambiente de campo rupestre da Chapada Diamantina, por exemplo, apenas 38,8\% é composta por espécies ornitófilas (MAchado et al. 2007) enquanto que, no presente estudo, este segmento representa $41,3 \%$ do total de espécies visitadas.

Em alguns ambientes, as espécies não-ornitófilas são exploradas em períodos de escassez de recursos, durante a estação mais seca (Brown \& Bowers 1985, Arizmendi \& Ornelas 1990, Fenster 1991, Buzato et al. 2000). Assim como observado por MACHADO et al. (2007), o uso deste tipo de recurso na caatinga não se limita à estação seca, ocorrendo ao longo do ano todo.

A habilidade de explorar recursos alimentares especializados e não especializados (plantas ornitófilas ou não), aliada à acentuada capacidade exploratória e de memorização de fontes de recursos (Pike 1978), tornam os beija-flores eficientes e, em muitos casos, versáteis em seu forrageio. O reflexo disto pode ser a polinização de um grande leque de espécies vegetais de ambiente semi-árido, tanto aquelas generalistas quanto aos vetores de pólen, quanto as mais especializadas em determinados grupos, sejam eles beija-flores ou insetos.

\section{AGRADECIMENTOS}

Ao CNPq, pelo suporte financeiro (processo 474217/20043), à UEFS e Prefeitura Municipal de Mucugê, pelo apoio logístico; ao Laboratório de Ornitologia e Mastozoologia/UEFS, pela infra-estrutura; a C.E.C. Nunes, M.C.B. Gomes, A.G. Coelho, S.S. Bastos, C.S. Santana, J.J. Almeida, A.D.C. Moura, S.C. Parente, D.M. Lima, M.H.B. Silveira e P.M.C. Gouvêa, pelos valorosos auxílios em campo; a L.P. Queiroz, F.A.R. Santos, F. França, A. Rapini, R.M. Harley, J.G. Jardim, M.N.S. Stapf, T. Senna, M.C. Assis, M.G.L. Wanderley, R. Louzada, A. Cortes, T.R.S. Silva, J. Semir e M.M.S. Castro, pelas identificações dos materiais botânicos; às biólogas e técnicas do HUEFS, pela importante assessoria; a N.M.R. Rodrigues, pela ajuda e incentivo; e aos revisores anônimos, pelas preciosas críticas e sugestões ao manuscrito. 


\section{LITERATURA CITADA}

Altmann, S.A. 1974. Observational study of behavior sampling methods. Behavior 49: 229-265.

Araujo, A.C. \& M. Sazima. 2003. The assemblage of flowers visited by hummingbirds in the capões of southern Pantanal, Mato Grosso do Sul, Brazil. Flora 198 (6): 427-435.

Arizmendi, M.C. \& J.F. Ornelas. 1990. Hummingbirds and their floral resources in a tropical dry forest in Mexico. Biotropica 22 (2): 172-180.

BAWA, K.S. 1990. Plant-pollinator interactions in tropical rain forests. Annual Review of Ecology and Systematics 21: 399-422.

Brown, J.H. \& M.A. Bowers. 1985. Community organization in hummingbirds: relationships between morphology and ecology. Auk 102: 251-269.

Buzato, S.; M. Sazima \& I. Sazima. 2000. Hummingbird-pollinated floras at three Atlantic Forest sites. Biotropica 32 (4b): 824841.

Conselho Brasileiro de Registros Ornitológicos. 2007. Lista primária de aves do Brasil. Available onliune at: http:// www.cbro.org.br/CBRO/index.htm [Accessed: 01/VI/2008]

Colaço, M.A.S.; R.B.S. Fonseca; S.M. Lambert; C.B.N. Costa; C.G. Machado \& E.L. Borba. 2006. Biologia reprodutiva de Melocactus glaucescens Buining \& Brederoo e M. pauscispinus G. Heinen \& R. Paul (Cactaceae), na Chapada Diamantina, Nordeste do Brasil. Revista Brasileira de Botânica 29 (2): 239-249.

FAEGRI, K. \& L. PIJL. 1980. The principles of pollination ecology. New York, Pergamon Press, 428p.

Feinsinger, P. 1983. Coevolution and pollination, p: 283-310. In: D. Futuyma \& M. Slatkin (Eds). Coevolution. Sunderland, Sinauer Associates Publishers, 376p.

FeinsingeR, P. \& R.K. CowelL. 1978. Community organization among neotropical nectar feeding birds. American Zoologist 18: 779795.

Fenster, C.B. 1991. Selection on floral morphology by hummingbirds. Biotropica 23 (1): 98-101.

Fischer, E.A. \& A.C. Araujo. 1995. Spatial organization of a bromeliad community in the Atlantic rainforest, southeastern Brazil. Journal of Tropical Ecology 11: 559-567.

Giulietti, A.M.; J.R. Pirani \& R.M. Harley. 1997. Espinhaço Range region, Eastern Brazil, p: 397-404. In: S.D. Davis; V.H. HeYwood; O. Herrera-MacBryde; J. Villa-lobos. \& A.C. Hamilton (Eds). Centers of plant diversity, a guide and strategy for their conservation. Oxford, Information Press, vol. 3, 582p.

Grantsau, R. 1988. Os beija-flores do Brasil. Rio de Janeiro, Ed. Expressão e Cultura, 233p.

JuncÁ, F.A.; L. Funch \& W. Rocha. 2005. Biodiversidade e conservação da Chapada Diamantina. Brasília, Ministério do Meio Ambiente, Série Biodiversidade 13, 435p.

Kaehler, M.; I.G. Varassin \& R. Goldenberg. 2005. Polinização em uma comunidade de bromélias em Floresta Atlântica
Alto-montana no Estado do Paraná, Brasil. Revista Brasileira de Botânica 28 (2): 219-228.

LEAL, F.C.; A.V. LoPes \& I.C. MAChado. 2006. Polinização por beijaflores em uma área de caatinga no Município de Floresta, Pernambuco, Nordeste do Brasil. Revista Brasileira de Botânica 29 (3): 379-389.

Machado, C.G. 2005. Aves, p: 357-375. In: F.A. Juncá; L. Funch \& W. Rосна (Eds). Biodiversidade e Conservação da chapada Diamantina. Brasília, Ministério do Meio Ambiente, Série Biodiversidade 13, 435p.

Machado, C.G. \& J. Semir. 2006. Fenologia da floração e biologia floral de bromeliáceas ornitófilas de uma área da Mata Atlântica do sudeste brasileiro. Revista Brasileira de Botânica 29 (1): 161-172.

Machado, C.G.; A.G. Coelho; C.S. Santana \& M. Rodrigues. 2007. Beija-flores e seus recursos florais em uma área de campo rupestre da Chapada Diamantina, Bahia. Revista Brasileira de Ornitologia 15 (2): 215-227.

Machado I.C.S. \& M. SAZIMA. 1995. Biologia da polinização e pilhagem por beija-flores em Ruelia asperula Lindau (Acanthaceae) na Caatinga de Pernambuco. Revista Brasileira de Botânica 18 (1): 27-33.

MaCHADO, I.C. \& A.V. Lopes. 2002. A polinização em ecossistemas de Pernambuco: uma revisão do estado atual do conhecimento, p: 583-596. In: M.Tabarelli \& J.M.C. Silva (Eds). Diagnóstico da biodiversidade de Pernambuco. Recife, Editora Massangana, 722p.

Machado, I.C. \& A.V. Lopes. 2003. Recursos florais e sistemas de polinização e sexuais em caatinga, p: 515-564. In: I.R. LEAL; M. Tabarelli \& J.M.C. Silva (Eds). Ecologia e conservação da Caatinga. Recife, Editora Universitária da UFPE, 822p.

Machado, I.C. \& A.V. Lopes. 2004. Floral Traits and Pollination systems in the Caatinga. Annals of Botany 94: 365-376.

MendonçA, L.B. \& L. Anjos. 2005. Beija-flores (Aves, Trochilidae) e seus recursos florais em uma área urbana do Sul do Brasil. Revista Brasileira de Zoologia 22 (1): 51-59.

Missouri Botanical Garden's Vast. 2007. VAScular Trópicos, W3Tropicos. Available online at: http://mobot.mobot.org/ W3T/Search/vast.html [Accessed: 10/X/2007]

Newstrom, L.E.; G.W. Frankie \& H.G. Baker. 1994. A new classification for plant phenology based on flowering patterns in lowland tropical rain forest trees at La Silva, Costa Rica. Biotropica 26 (2): 141-159.

Parrini, R.; M.A. Raposo; J.F. Pacheco; A.M.P. Carvalhaes; T.A. Melo-Junior; P.S.M. Fonseca \& J. Minns. 1999. Birds of the Chapada Diamantina, Bahia, Brazil. Cotinga 11: 86-95.

PIKE, G.H. 1978. Optimal foraging in hummingbirds: testing the marginal value theorem. American Zoologist 18: 739752.

Poulin, B.; G. Lefebvre \& R. McNeil. 1992. Tropical avian phenology in relation to abundance and exploitation of food resources. Ecology 73: 2295-2309.

SAZIMA I.; S. BuZATO \& M. SAZIMA. 1996. An assemblage of humming- 
Obird-pollinated flowers in a montane forest in southeastern Brazil. Acta Botanica Brasilica 109: 149-160.

Silva, J.M.C; M.A. Souza; A.G.D. Bieber \& C.J. Carlos. 2003. Aves da Caatinga: status, uso do habitat e sensitividade, p: $237-$ 273. In: I.R. LeaL; M. Tabarelli \& J.M.C. Silva (Eds). Ecologia e conservação da Caatinga. Recife, Editora Universitária da UFPE, 822p.

Sneath, P.H.A. \& R.R. SoKal. 1973. Numerical taxonomy. The principles and practice of numeral classification. San Francisco, Freeman Press, 573p.

Snow, D.W. \& B.K. SNow. 1986. Feeding ecology of hummingbirds in the Serra do Mar, southeastern Brazil. El Hornero 12: 286-296

Snow, D.W. \& D.I. Teixeira. 1982. Hummingbirds and their

Submitted: 07.VI.2008; Accepted: 11.VI.2009.

Editorial responsibility: Paulo Inácio López de Prado flowers in the coastal mountains of southeastern Brazil. Journal of Ornithology 123: 446-450.

STILES, F.G. 1981. Geographical aspects of bird-flower coevolution, with particular reference to Central America. Annals of Missouri Botanical Garden 68: 323-351.

Stradmann, M.P.S. 1998. Plano de Manejo - Parque Municipal de Mucugê. Mucugê, Projeto Sempre-Viva/ MMA/PNMA/ PED 96CV00027/96, 423p.

Temeles, E.J. \& Y.B. Linhart. 2002. The role of Flower Windth in Hummingbird Bill Lenght-Flower Length Relationships. Biotropica 34 (1): 68-800.

VASCONCELOS, F.V \& J.A. LOMBARDI. 2001. Hummingbirds and their flowers in the campos rupestres of southern Espinhaço Range, Brazil. Melopsittacus 4 (1): 3-30. 